\title{
Subject Index to Volume 21
}

Acetoacetyl coenzyme A thiolase, deficiency, ketotic hypoglycemia with, 211

Acidemia

D-glyceric, fructose metabolism and, 502

metabolic, vasopressin and catecholamine secretion during, ovine fetus, 38

Acquired immunodeficiency syndrome, pediatric, HTLV-III neutralizing antibody related to, 547

Actomyosin, maturational changes and, arterial and venous tissues, dog, 152

Adolescence, proteinuria in, characterization of, 442

Age, stimulation by Escherichia coli and, effect on intestinal guanylate cyclase activity, 551

Airway obstruction, upper, effects of hyperoxia on arousal response to, neonatal lamb, 116

Albumin, synthesis, premature infant, 49

Amino acid

branched chain, carbon and nitrogen arteriovenous concentration differences, ovine fetal hindlimb, 44

plasma differences, human milk versus cow milk, very low birth weight infant, 301

Amniotic fluid, 1,25-hydroxycholecalciferol receptor content, 432

Anemia

effect on thermogenic response to environmental cold stress, newborn piglet, 482

ouabain effects on oxygen physiology, neonatal lamb, 447

postnatal, regulation of erythropoiesis, suckling rabbit, 1

Apolipoprotein B, measurement, variables, neonate, 608

Artery, maturation, pharmacological characteristics and actomyosin content, dog, 152

Asphyxia, catecholamine response, indomethacin effect, neonatal piglet, 534

Autoradiography, regional cerebral blood flow determined by, immature rat, 471

Basolateral membrane, calcium transport by, developmental changes, 257

B-Cell line, Epstein-Barr virus-transformed, from severe combined immunodeficiency patient, 331

Behavior disorder, purine nucleoside phosphorylase deficiency and, case report, 137

Bicarbonate, uptake, sweat gland, cystic fibrosis, 79

Bile acid

biliary composition, early gestation, human fetus, 197

metabolism, during development, fetal liver, 99

uptake, acinar gradient for, developing rat liver, 417

Biliary tract, ontogeny, postnatal changes, guinea pig, 170

\section{Bilirubin}

lumirubin formation from, fatty acid enhancement, 530

production and excretion, effect of tin (IV)-

Birth protoporphyrin-IX, rat, 487

catecholamine surge, effect of naloxone, newborn lamb, 590

fetal to newborn transition, renal hemodynamics and functional changes during, 229

Blood

chemistry, normal human fetus, midtrimester of pregnancy, 579

intraventricular, effect on cerebral blood flow, newborn puppy, 511

Blood flow

brain, effect of prolonged hypercarbia, awake newborn piglet, 29

cerebral

effect of intraventricular blood, newborn puppy, 511

external high frequency oscillation effects, monkey, 166

gastrointestinal, oxygen consumption and, neonatal piglet, 93

regional, effects of dopamine, neonatal lamb, 131

regional cerebral

autoradiographic determination, immature rat, 471

theophylline effect on hypoxia response, newborn piglet, 573

Brain

blood flow, effect of prolonged hypercarbia, awake newborn piglet, 29

energy state, hypotension effect during neonatal seizure, dog, 357

trisomic, superoxide metabolism in, fetal mice and human fibroblasts, 88

Breathing

inspiratory resistive loading effect, crural and costal diaphragm electromyogram, piglet, 25

onset of, surfactant secretion, newborn rat lung, 5

Burst promoting activity, production, by cord blood, 285

Caffeine

chronic ingestion, effect on myocardial function, fetal and neonatal rat, 391

renal effect, newborn rabbit, 615

Calcium, intestinal transport, basolateral membrane, suckling and adolescent rats, 257

Carbon, branched chain amino acid arteriovenous concentration differences, ovine fetal hindlimb, 44

Carbon dioxide, production, open-circuit system measurement evaluated, neonate, 58

Cardiac output, effect of prolonged hypercarbia, awake newborn piglet, 29

Carnitine, renal handling, selective tubulopathy and Fanconi syndrome, 201

Casein hydrolysate, pooled human milk supplementation, very low birth weight infant, 458
Catecholamine

birth surge, effect of naloxone, newborn lamb, 590

secretion, during metabolic acidemia ovine fetus, 38

C3bi receptors, surface membrane expression, neonatal neutrophils, 306

Chest wall, compression, high frequency, normal lungs, cat, 183

Chloride, deficiency, canine puppy, 497

Choledochoduodenal junction, motility, agedependent changes, guinea pig, 170

Cholesterol, early feeding, effect on intestinal lipid uptake, 347

Circulation

cerebral, indomethacin effect, neonatal piglet, 188

leukotriene $\mathrm{C}_{4}$ and $\mathrm{D}_{4}$ effects, neonatal lamb, 176

Citrate, urinary excretion, glycogenosis type I, 279

Cockayne syndrome, ultraviolet survival in, 34

Collagen, concentration, dexamethasone effect, perinatal rat lung, 603

Compression, chest wall, high frequency, normal lungs, cat, 183

Cord blood

mononuclear cells, low burst promoting activity production by, 285

neutrophils, kinetics of superoxide anion production in, 205

Cyclic adenosine monophosphate, $\beta$ receptors and, fetal lung, 142

Cystic fibrosis

role of band 3 protein, kidney and other tissues, 235

sweat gland, lactate and bicarbonate uptake, 79

sweat gland duct cells, doming by, 72

Cystine

transport

isolated jejunal epithelium, cystinuric patients, 477

lysosomal, cystinosis variants and their parents, 193

Cystinosis, nephropathic, lysosomal cystine transport in, 193

Cystinuria, cystine flux, in jejunal epithelium, 477

Dehydrogenation disorder, multiple acylCoA, clinical and biochemical variation, 371

Dexamethasone, effects on growth, elastin concentration, and collagen concentration, perinatal rat lung, 603

Diabetes, maternal, effect on postnatal development of liver mitochondria, rat, 266

Dialysis, peritoneal, osteocalcin clearance by, end-stage renal disease, 296

Diamine oxidase

effect of hydrocortisone, thyroxine, and phenobarbital on

newborn rat intestine, 368

Diaphragm

costal, electromyogram activity, compared with crural, piglet, 25 
electromyogram spectral analysis, during hypoxemia, infant monkey, 238

resistive loading response, neonatal piglet, 121

Diet, high-cholesterol, intestinal permeability to lipids enhanced by, rabbit, 347

Diplegia, spastic, purine nucleoside phosphorylase deficiency and, case report, 137

Docosahexaenoic acid, supplementation, effect on fatty acid content of red cell membranes, preterm infants, 507

Dolichols, intestinal, developing small intestine, rat, 261

Dopamine, infusion, regional blood flow effects, neonatal lamb, 131

Ductus venosus, prostaglandin $E_{1}$ effect, newborn lamb, 219

Elastin, concentration, dexamethasone effect, perinatal rat lung, 603

Electromyogram

diaphragm, infant monkey, 238

inspiratory resistive loading effect, costal and crural diaphragm, piglet, 25

spectral analysis of during hypoxemia, infant monkey, 235

Endotoxin, hyperoxia and, neonatal rat, 109 Energy

expenditure

assessment by doubly labeled water method, infants, 242

day to day variability, low birth weight neonate, 66

Enzyme, mucosal, breast milk effect, small intestine, neonatal rabbit, 126

Epinephrine, racemic, effect on ventilatory function after extubation, neonate, 381

Erythroblastopenia, transient, erythrocyte aging and band 4.1, 275

Erythrocyte

aging, membrane protein 4.1 and, 275

lipid abnormalities, Reye's syndrome, 352

Erythropoiesis

regulation, with and without postnatal anemia, suckling rabbit, 1

stimulating factors, dose response and chromatographic studies, neonatal mouse, 148

Escherichia coli, heat-stable enterotoxin, effect on intestinal guanylate cyclase activity, 551

Estrogen, serum levels, respiratory distress syndrome and, neonate, 386

Exercise, ventilation and $\mathrm{CO}_{2}$ production coupling during, children, 568

Extubation, ventilatory function after, effect of epinephrine, neonate, 381

Fanconi syndrome, renal handling of carnitine, 201

Fatty acid

effect on albumin-bound bilirubin, 530

red blood cell membranes, effect of fish oil supplementation, preterm infants, 507

Feeding, effects on gastrointestinal blood flow and oxygen consumption, hypoxemic neonatal piglet, 93

Feeding regimens, metabolic effects, isocaloric total parenteral nutrition solutions compared, 538

bile acid metabolism during development, human liver, 99

blood chemistry, midtrimester of pregnancy, 579 branched-chain amino acid carbon and nitrogen arteriovenous concentration differences, ovine fetal hindlimb, 44

early gestation, biliary bile acid composition, 197

lung

development, guinea pig, 427

maturation, hyperinsulinemic rat, 436

$\beta$ receptors and cyclic adenosine monophosphate receptors in, 142

surfactant pool alterations, rat, 5

metabolic acidemia, vasopressin and catecholamine secretion during, lamb, 38

myocardial function, effect of chronic caffeine intake on, rat, 391

renal function, effects of delivery on, sheep, 229

thyroidectomy, neonatal thermogenesis affected by, lamb, 453

vitamin D-dependent calcium-binding proteins in, 362

Fish oil, supplementation, effect on fatty acid content of red cell membranes, preterm infant, 507

Formula, cow milk, plasma amino acids, compared with human milk, very low birth weight infant, 301

Fructose, metabolism, D-glyceric acidemia associated with, 502

Gestation, early, biliary bile acid composition, fetus, 197

Glucose

intestinal transport, physiologic role of epidermal growth factor, suckling rat, 404

intolerance, young female rats exposed to fetal hyperinsulinemia, 83

role in intestinal glucose transport, suckling rat, 404

Glutamine, oxidation, small intestine, suckling rat, 214

Glycerol, urinary excretion, glycogenosis type I, 279

Granulocyte, group B streptococcal toxin and, pulmonary response, lamb, 159

Growth and development

abnormal glucose tolerance and, young female rats exposed to fetal hyperinsulinemia, 83

myocardial function, chronic caffeine intake effects, fetal and neonatal rat, 391

respiratory control maturation, during exercise, 568

Guanylate cyclase, small and large intestinal activity, effect of age and Escherichia coli stimulation, 55

Heart

congenital malformation, research advances, 219

function, after caffeine intake, fetal and neonatal rat, 391

Hematocrit, maternal hemodynamics and, late-pregnant guinea pig, 584

Hemodilution, isovolemic, hemodynamic effects, late-pregnant guinea pig, 584

Hemolytic uremic syndrome, neutrophil function, rabbit, 252

Hepatocyte, respiratory characteristics, neonatal rat, 492

Hirschsprung's disease, neural cell migration and, experimental study, chicken embryo, 466

HTLV-III, neutralizing antibody, pediatric acquired immunodeficiency related to, 547
Human milk

casein hydrolysate supplementation, very low birth weight infant, 458

macrophage function, lactoferrin and, 54

plasma amino acids, compared with cow milk formula, very low birth weight infant, 301

Hyaline membrane disease, high frequency ventilation, infant baboon, 594

Hydrocortisone, effect on diamine oxidase activity, newborn rat intestine, 368

1,25-Hydroxycholecalciferol, specific receptors, human amniotic cells, 432

Hypercarbia, prolonged, effect on brain blood flow and cardiac output, awake newborn piglet, 29

Hyperglycemia, combined with hypoxiaischemia, cerebral metabolic responses, immature rat, 524

Hyperinsulinemia

fetal

growth and glucose tolerance in young female rats exposed to, 83

lung maturation and, rat, 436

Hyperoxia

effects on arousal response, upper airway obstruction, neonatal lamb, 116

endotoxin treatment and, neonatal rat, 109

neonatal exposure, vascular pulmonary dysplasia and, rat, 14

Hypoglycemia, ketotic, acetoacetyl coenzyme A thiolase deficiency and, 211

Hypotension, effect on brain energy state, prolonged neonatal seizure, dog, 357

Hypothermia, intestinal ischemic injury after, neonatal piglet, 422

Hypoxemia

electromyogram spectral analysis during, diaphragm, infant monkey, 238

feeding and, effects on gastrointestinal blood flow and oxygen transport, newborn piglet, 93

Hypoxia

neonatal, effect on postnatal development of liver mitochondria, rat, 266

regional cerebral blood flow response, theophylline effect, newborn piglet, 573

Hypoxia-ischemia, cerebral metabolic responses, hyperglycemic immature rat, 524

Immunodeficiency, severe combined, B-cell line from, lymphoma patient, 331

Immunoglobulin

group B streptococcus treatment, alone and in combination with penicillin $G$ 289

ribavirin and, respiratory syncytial virus treatment, cotton rat, 270

Indomethacin

effect on catecholamine response to asphyxia, neonatal piglet, 534

effect on cerebral circulation, neonatal piglet, 188

Infants

energy expenditure assessment, doubly labeled water method validity, 242

hypoxemia, electromyogram spectral analysis, diaphragm, monkey, 238

hyaline membrane disease, high frequency ventilation, infant baboon, 594

sleep state determination, respiratory variability used for, 556

Insulin, effect on leucine kinetics, maple syrup urine disease, 10

Intestine

ischemic injury, after mild hypothermic 


\section{SUBJECT INDEX TO VOLUME 21}

stress, neonatal piglet, 422

neonatal, effect of hydrocortisone, thyroxine, and phenobarbital on diamine oxidase activity, rat, 368

Iron, supplementation, erythropoiesis regulation, suckling rabbits with and without postnatal anemia, 1

Irradiation, ultraviolet, Cockayne syndrome diagnosis, 34

Isoprenolog, distribution, developing small intestine, rat, 261

Jejunum, epithelium, cystine fluxes across, cystinuric patients, 477

Kidney

band 3 protein immunocytochemistry, cystic fibrosis patients, 235

carnitine handling, selective tubulopathy and Fanconi syndrome, 201

chronic insufficiency, lipid-lipoprotein abnormalities, 462

end-stage disease, osteocalcin clearance by peritoneal dialysis, 296

growth arrest, partial ureteral obstruction and, neonatal guinea pig, 338

renal function and hemodynamics, effects of delivery on, sheep, 229

theophylline and caffeine effects, newborn rabbit, 615

Lactate

uptake, sweat gland, cystic fibrosis, 79

urinary excretion, glycogenosis type I, 279

Lactoferrin

activator of thymidine incorporation into DNA, rat crypt cells, 563

inhibition of prostaglandin $E_{2}$ secretion, breast milk macrophages, 54

Large intestine, guanylate cyclase activity, effect of age and Escherichia coli stimulation, 551

Leucine, kinetics, insulin effect, maple syrup urine disease, 10

Leukocyte

polymorphonuclear

adherence and chemotaxis after proteincalorie malnutrition, neonatal rat, 542

effect of group B streptococcal type III antigen, 517

Leukotriene $\mathrm{C}_{4}$, effect on circulation, compared with leukotriene $\mathrm{D}_{4}$, neonatal lamb, 176

Leukotriene $\mathrm{D}_{4}$, effect on circulation, compared with leukotriene $\mathrm{C}_{4}$, neonatal lamb, 176

Lipid

abnormalities

chronic renal insufficiency, 462

erythrocyte, in Reye's syndrome, 352

intestinal uptake, high-cholesterol diet and, rabbit, 347

Lipoprotein, abnormalities, chronic renal insufficiency, 462

Lithocholic acid, metabolism, during devel-

Liver opment, fetal liver, 99

mitochondria, postnatal development, neonatal rat, 266

neonatal, bile acid uptake, rat, 417

Loading, resistive, response, neonatal piglet, 121

Lumirubin, formation, fatty acid enhancement, 530

Lung

development, surfactant, morphology, pre- mature viability, fetal guinea pig, 427

fetal, surfactant pool alterations, rat, 5

group B streptococcal toxin and, granulocyte role, lamb, 159

growth, elastin concentration, and collagen concentration, dexamethasone effects, perinatal rat, 603

maturation, hyperinsulinemic rat fetus, 436

neonatal, surfactant pool alterations, rat, 5

normal, high frequency chest wall compression and, cat, 183

pulmonary function, sick newborn infant, 313

$\beta$ receptors and cyclic adenosine monophosphate in, human fetus, 142

vascular pulmonary dysplasia, neonatal hyperoxia and, rat, 14

Luteinizing hormone, bioactive, pulsatile release, prepubertal girls, 409

Lysosome, cystine transport, cystinosis variants and their parents, 193

Macrophage, human milk, lactoferrin effect, 54

Macrosomia, fetal, glucose intolerance and, young female rat, 83

Malnutrition, protein-calorie, polymorphonuclear leukocyte adherence and chemotaxis after, neonatal rat, 542

Maple syrup urine disease, leucine kinetics, insulin effect, 10

Maturation

arterial and venous tissue, pharmacological characteristics, dog, 152

choledochoduodenal junction motility, postnatal changes, guinea pig, 170

intestinal, calcium transport, basolateral membranes, suckling and adolescent rats, 257

Milk

epidermal growth factor-deficient, intestinal glucose transport, suckling rat, 404

human, effect on mucosal enzymes, small intestine, neonatal rabbit, 126

Mitochondria, liver, postnatal development, neonatal rat, 266

Mononuclear cells, cord blood, low burst promoting activity production by, 285

Morphogenesis, cardiac, research advances, 219

Motility, biliary, postnatal changes, guinea pig, 170

Myocardium, function, effect of chronic caffeine intake, fetal and neonatal rat, 391

Naloxone, effect on catecholamine surge at birth, newborn lamb, 590

Neonate

adaptation, effect of naloxone on catecholamine surge, lamb, 590

anemic, thermogenic response to environmental cold stress and, piglet, 482

apolipoprotein B measurement, variables, 608

bile acid uptake, developing liver, rat, 417

blood flow response to hypoxia, theophylline effect, piglet, 573

catecholamine response to asphyxia, indomethacin effect, neonatal piglet, 534

circulation

cerebral, indomethacin effects, piglet, 188

differential effects of leukotriene $\mathrm{C}_{4}$ and
$\mathrm{D}_{4}$, lamb, 176

ductus venosus, prostaglandin $E_{1}$ effect, lamb, 219

hepatocyte, respiratory characteristics, rat, 492

hyperoxia effect

arousal response to upper airway obstruction, lamb, 116

vascular pulmonary dysplasia and, rat, 14

hypotension effect, during prolonged seizure, dog, 357

hypothermic stress, intestinal ischemic injury after, piglet, 422

low birth weight, energy expenditure variability, day to day, 66

lung, surfactant pool alterations, rat, 5

mucosal enzyme activity, effect of breast milk, small intestine, neonatal rabbit, 126

myocardial function, effect of chronic caffeine intake on, rat, 391

neutrophil

count and aggregation, effect of group B streptococcus, 326

effects of neutrophil migration inhibitory factors on, 377

periodicity cycle length, progressive shortening, 247

surface membrane expression of $\mathrm{C} 3 \mathrm{bi}$ receptors, 306

oxygen consumption and carbon dioxide production, open-circuit system measurement evaluated, 58

oxygen physiology, ouabain effects, lamb, 447

oxygen toxicity, endotoxin treatment effect, rat, 109

partial ureteral obstruction, renal growth arrest, guinea pig, 338

phototherapy, transepithelial electric potential difference in, 279

plasma erythropoiesis stimulating factors in, dose response and chromatographic studies, mouse, 148

platelet, contractile activity, 293

protein-calorie malnutrition, polymorphonuclear leukocyte adherence and chemotaxis after, neonatal rat, 542

regional blood flow, effects of dopamine infusion, lamb, 131

resistive loading, response, neonatal piglet, 121

serum estrogen levels, respiratory distress syndrome related to, 386

severe chloride deficiency, canine puppy, 497

sick, pulmonary function, 313

skin maturation, electrical skin resistance related to, 21

thermogenesis, fetal thyroidectomy effect, lamb, 453

ventilatory function after extubation, effect of racemic epinephrine, 381

very low birth weight

casein hydrolysate supplementation, 458

plasma amino acid differences, human milk versus cow milk, 301

Neural cell, migration, Hirschsprung's disease and, chicken embryo, 466

Neural crest, ablation, congenital heart defects and, 219

Neutrophil

aggregation, induced by group B streptococcal extract,neonatal lamb, 326

cord blood, kinetics of superoxide anion production in, 205

count, reduced by group B streptococcal 


\section{SUBJECT INDEX TO VOLUME 21}

extract, neonatal lamb, 326

function, hemolytic uremic syndrome, rabbit, 252

migration inhibitory factors, effects on neonatal neutrophils, 377

neonatal, surface membrane expression of C3bi receptors, 306

Nitrogen

branched chain amino acid arteriovenous concentration differences, ovine fetal hindlimb, 44

retention, isocaloric intravenous nutritional regimens compared, 538

Nutrition

casein hydrolysate supplementation, pooled human milk, very low birth weight infant, 458

early, intestinal lipid uptake and, 347

total parenteral

effect of energy source, 538

taurine concentrations, plasma, blood cells, urine, 399

Obstruction

upper airway, effects of hyperoxia on arousal response, neonatal lamb, 116

ureteral, renal growth arrest and, neonatal guinea pig, 338

Oscillation, external high frequency, cerebral blood flow affected by, monkey, 166

Osteocalcin, clearance, peritoneal dialysis, end-stage renal disease, 296

Ouabain, effects on oxygen physiology, anemic neonatal lamb, 447

2-Oxoglutarate, urinary excretion, glycogenosis type I, 279

Oxygen

consumption

gastrointestinal blood flow and, neonatal piglet, 93

open-circuit system measurement evaluated, neonate, 58

physiology, ouabain effects, anemic lamb, 447

toxicity, endotoxin treatment effect, neonatal rat, 109

Patent ductus arteriosus, atrial natriuretic peptide in, 396

Penicillin G, group B streptococcus treatment, alone and in combination with human immunoglobulin, 289

Peptide, atrial natriuretic, in patent ductus arteriosus, 396

Periodicity, cycle length, progressive shortening, normal infant, 247

Phenobarbital, effect on diamine oxidase activity, newborn rat intestine, 368

Phototherapy, transepithelial electric potential difference, neonate, 279

Placenta, term, effect of tetrahydrocannabinol, 104

Platelet, contractile activity, neonate, 293

Pregnancy

hematocrit and maternal hemodynamics related, late-pregnant guinea pig, 584

midtrimester, blood chemistry of normal human fetus, 579

Prematurity

albumin synthesis, turnover determined with $\left[{ }^{15} \mathrm{~N}\right]$ Glycine, 49

fish oil supplementation, effect on fatty acid content of red blood cell membranes, 507

Prostaglandin, concentration, after intestinal protein hypersensitivity, small intestinal mucosa, rat, 414

Prostaglandin $E_{1}$, ductus venosus opened by, newborn lamb, 219

Prostaglandin $E_{2}$, secretion, lactoferrin inhibition, breast milk macrophages, 54

Protein

band 3, immunocytochemistry, kidney, cystic fibrosis patients, 235

calcium-binding, vitamin D-dependent, human fetus, 362

intestinal hypersensitivity, effect on prostaglandin concentration, small intestinal mucosa, rat, 414

Proteinuria, recumbent, ambulatory, and postexercise, characterization in the adolescent, 442

Puberty, luteinizing hormone pulse characteristics, compared with prepubertal girls, 409

Purine nucleoside phosphorylase, deficiency, enzymologic, clinical, and immunologic characteristics, 137

Respiration

control maturation

during exercise, children, 568

hepatocyte, neonatal rat, 492

periodicity cycle length, progressive shortening, normal infants, 247

pulmonary function, sick newborn infant, 313

resistive loading response, neonatal piglet, 121

variability, sleep state determined by, infants, 556

ventilatory function after extubation, effect of epinephrine, neonate, 381

Respiratory distress syndrome, serum estrogen and, neonate, 386

Reye's syndrome, erythrocyte lipid abnormalities in, 352

Ribavirin, immunoglobulin and, respiratory syncytial virus treatment, cotton rat, 270

Seizure, neonatal, hypotension during, dog, 357

Skin, maturation, electrical skin resistance

Sleep state, determination, respiratory variability used for, infant, 556

Small intestine

glutamine oxidation by, suckling rat, 214

guanylate cyclase activity, effect of age and Escherichia coli stimulation, 551

mucosal enzyme activity, breast milk effect, neonatal rabbit, 126

postnatal development, dolichol content and isoprenolog distribution, rat, 261

rise of prostanoids in, after intestinal protein hypersensitivity, 414

Streptococcus

group B related to, neonate, 21 granulocyte role in pulmonary response to, lamb, 159

human immunoglobulin and penicillin $G$ treatment efficacy, 289

neutrophil count reduced by, neonatal lamb, 326

type III antigen, effect on polymorphoStress nuclear leukocyte function, 517

environmental cold, thermogenic response, anemic newborn piglet, 482

hypothermic, intestinal ischemic injury after, neonatal piglet, 422

Superoxide, metabolism, trisomic brain, feta mice and human fibroblasts, 88

Superoxide anion, production kinetics, cord blood neutrophils, 205

Surfactant, onset of breathing, fetal and newborn rat lung, 5

Sweat gland

lactate and bicarbonate uptake, cystic fibrosis and normal subjects, 79

tissue culture, alterations in dome formation, cystic fibrosis, 72

Taurine, concentration in plasma, blood cells, and urine, total parenteral nutrition patients, 399

Tetrahydrocannabinol, effect on $\alpha$-amino isobutyric acid uptake, human placental slices, 104

Theophylline, renal effect, newborn rabbit, 615

Thermogenesis, neonatal, effect of fetal thyroidectomy on, lamb, 453

Thymidine, incorporation into DNA, lactoferrin stimulation, 563

Thyroidectomy, fetal, neonatal thermogenesis affected by, lamb, 453

Thyroxine, effect on diamine oxidase activity, newborn rat intestine, 368

Tin (IV)-protoporphyrin IX, effect on bilirubin production and excretion, rat, 487

Tubulopathy, selective, renal handling of carnitine and, 201

Ultraviolet, irradiation, Cockayne syndrome diagnosis, 34

Ureter, partial obstruction, renal growth arrest and, neonatal guinea pig, 338

Vasopressin, secretion, during metabolic acidemia, ovine fetus, 38

Vein, maturation, pharmacological characteristics and actomyosin content, dog, 152

Ventilation

high-frequency, hyaline membrane disease, infant baboon, 594

negative, external high frequency oscillation and cerebral blood flow, monkey, 166

Virus, respiratory syncytial, immunoglobulin and ribavirin efficacy, cotton rat, 270

Vitamin D, role in cellular differentiation and growth, 362

Water, doubly labeled, energy expenditure assessment method, infant, 242 